\title{
Effect of Some Geometric Transfers on Homology Groups
}

\author{
Samy M. Mostafa ${ }^{1}$, Abdelaziz E. Radwan ${ }^{2}$, Fayza Abelhalim Ibrahem ${ }^{2}$ \& Fatema F.Kareem ${ }^{2,3}$ \\ ${ }^{1}$ Department of Mathematics, Faculty of Education, Ain Shams University, Roxy, Cairo, Egypt 。 \\ ${ }^{2}$ Department of Mathematics, Faculty of science, Ain Shams University, Cairo, Egypt. \\ ${ }^{3}$ Department of Mathematics, Ibn-Al-Haitham college of Education, University of Baghdad, Iraq. \\ Correspondence: Fatema F.Kareem, Department of Mathematics, Faculty of science, Ain Shams University, \\ Cairo, Egypt. E-mail: fa_sa20072000@yahoo.com
}

$\begin{aligned} & \text { Received: September 17, } 2014 \\ & \text { Accepted: October 21, } 2014 \quad \text { Online Published: October 30, } 2014 \\ & \text { doi:10.5539/jmr.v6n4p90 }\end{aligned}$ URL: http://dx.doi.org/10.5539/jmr.v6n4p90

\begin{abstract}
In this work, we introduce the results of some geometric transformation of the manifold on the homology group. Some types of folding and unfolding on a wedge sum of manifolds which are determined by their homology group are obtained. Also, the homology group of the limit of folding and unfolding on a wedge sum of 2manifolds is deduced.
\end{abstract}

Keywords: manifolds, homology group, folding, unfolding.

Mathematics Subject Classification: 51H20, 57N10, 57T10.

\section{Introduction}

The notion of folding on manifolds has been introduced by (Robertson, 1977). The conditional folding of manifold and a graph folding have been defined by (El-Kholy, 1981-2005). Also, the unfolding of a manifold has been defined and discussed by (M.El-Ghoul, 1988). Many authors have studied the folding of many types of manifolds. The homology groups of some types of a manifold are discussed by (M.El-Ghoul, 1990; M.Abu-Saleem, 2010). (Abu-Saleem, 2007) introduced the results of some geometric transformation of the manifold on the fundamental group. In this paper, we introduce the folding and unfolding of some types of manifolds which are determined by their homology group and we study and discuss the homology group of the limit of folding and unfolding on a wedge sum of 2-manifolds.

\section{Preliminaries}

In this section, we introduce some necessary definitions which are needed especially in this paper.

Definition 2.1 The $n$-dimension manifold is a Hausdorff space such that each point has an open neighborhood homeomorphic to the open n-dimensional $\operatorname{disc} U_{n}=\left\{x \in R^{n}:|x|<1\right\}$, where $n$ is positive integer (W. S. Massey, 1976).

Definition 2.2 An abstract simplicial complex is a pair $X=(V, S)$ where $V$ is a finite set whose elements are called the vertices of $X$ and $S$ is a set of non-empty subsets of $V$. Each element $s \in S$ has precisely $n+1$ elements $(n \geq 0), s$ is called an $n$-simplex. (Thus an abstract simplex is merely the set of its vertices). The simplexes of $X$ satisfy the following conditions;

(1) $v \in V \Rightarrow\{v\} \in S$;

(2) $s \in S, t \subset s, t \neq \phi \Rightarrow t \in S$.

The dimension of $S$ is $n$ and the dimension of $X$ is the largest of the dimensions of its simplexes (P.J. Giblin, 1977).

Definition 2.3 Let $s=\left[v^{0}, \ldots, v^{n}\right]$ be an oriented $n$-simplex of $S$ for some $n>0$. The boundary homomorphism $\partial_{n}$ of $s$ is $(n-1)$-chain

$\partial_{n}\left[v^{0}, \ldots, v^{n}\right]=\sum_{i=0}^{n}(-1)^{i}\left[v^{0}, \ldots, v^{j-1}, v^{j+1}, \ldots, v^{n}\right]$ i.e. $\partial_{n}: C_{n} \rightarrow C_{n-1}$ and for $n=0, \partial_{0}$ is the null function (P.J. Giblin, 
Definition 2.4 The sequence $\quad \cdots \stackrel{\partial_{n+2}}{\longrightarrow} C_{n+1}(S) \stackrel{\partial_{n+1}}{\longrightarrow} C_{n}(S) \stackrel{\partial_{n}}{\longrightarrow} \cdots \stackrel{\partial_{2}}{\longrightarrow} C_{1}(S) \stackrel{\partial_{1}}{\longrightarrow} C_{0}(S)$ is called the chain complex of $S$. For any $n, \partial_{n} \circ \partial_{n+1}=0$. In the sequence, $\operatorname{ker} \partial_{n}$ is denoted by $Z_{n}(S)$, and elements of $Z_{n}(S)$ are called $n$-cycles. Also, $\operatorname{Im} \partial_{n+1}$ is denoted by $B_{n}(S)$, and elements of $B_{n}(S)$ are called $n$-boundaries. And since $B_{n} \subset Z_{n}$ there is a quotient group $H_{n}=Z_{n} / B_{n}$, called the $n$-th homology group of $S$ (P.J. Giblin, 1977).

Notation: $H_{n}(S)$ is measure the number of independent $n$-dimensions of holes in $S$, where $0 \leq n \leq \operatorname{dim} S$.

Definition 2.5 Let $M$ and $N$ be two manifolds of dimensions $m$ and $n$ respectively. A map $f: M \rightarrow N$ is said to be an isometric folding of $M$ into $N$ if, for every piecewise geodesic path $\gamma: I \rightarrow M,(I=[0,1] \subseteq R)$, the induced path $f \circ \gamma: I \rightarrow N$ is piecewise geodesic and of the same length as $\gamma$. If $f$ does not preserve length, it is called a topological folding (E.El-Kholy, 1981; S.A.Robertson, 1977).

Definition 2.6 Let $M$ and $N$ be two manifolds of the same dimensions. A map $g: M \rightarrow N$ is said to be unfolding of $M$ into $N$ if, for every piecewise geodesic path $\gamma: I \rightarrow M$, the induced path $g \circ \gamma: I \rightarrow N$ is piecewise geodesic with length greater than $\gamma$ ( M.El-Ghoul, 1988).

Definition 2.7 Let $M$ and $N$ be two manifolds of the same dimensions and unf $: M \rightarrow M^{\prime}$ be any unfolding of $M$ into $M^{\prime}$. Then, a map $\overline{u n f}: H_{n}(M) \rightarrow H_{n}\left(M^{\prime}\right)$ is said to be an induced unfolding of $H_{n}(M)$ into $H_{n}\left(M^{\prime}\right)$ if

$\overline{u n f}\left(H_{n}(M)\right)=H_{n}(u n f(M)) \quad$ (M.Abu-Saleem, 2010).

Definition 2.8 Let $\mathrm{X}$ and $\mathrm{Y}$ be spaces, and choose points $x_{0} \in X, y_{0} \in Y$ in each space. The wedge sum $X \vee Y$ is the quotient of the disjoint union $X \cup Y$ obtained by identifying $x_{0}$ and $y_{0}$ to a single point $X \vee Y=X \bigcup_{x_{0} \sim y_{0}} Y \approx(X \coprod Y) / x_{0} \sim y_{0}$ (A.Hatcher, 2001, $\underline{\text { http://www.math.coronell.edu/hatcher }}$ ).

\section{The Main Results}

In this section, we will introduce the following:

Lemma 3.1 The homology group $H_{n}\left(S^{2}\right)$ of any folding of $S^{2}$ is either isomorphic to $Z$ or identity group.

Proof. First, for folding with singularity of $S^{2}$ as in Figure 1(a) then $H_{0}\left(F\left(S^{2}\right)\right) \approx \mathrm{Z}$ and for $n>0, H_{n}\left(F\left(S^{2}\right)\right)=0$. Also, folding without singularity of $S^{2}$ is a manifold homeomorphic to $S^{2}$ as in Figure $1(\mathrm{~b})$ and so $H_{0}\left(F\left(S^{2}\right)\right) \approx \mathrm{Z}, H_{1}\left(F\left(S^{2}\right)\right)=0, H_{2}\left(F\left(S^{2}\right)\right) \approx \mathrm{Z}$ and for $n>2$, $H_{n}\left(F\left(S^{2}\right)\right)=0$

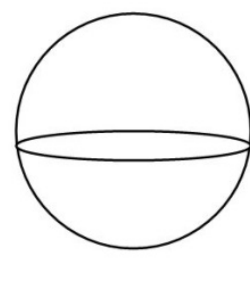

Folding with singularity
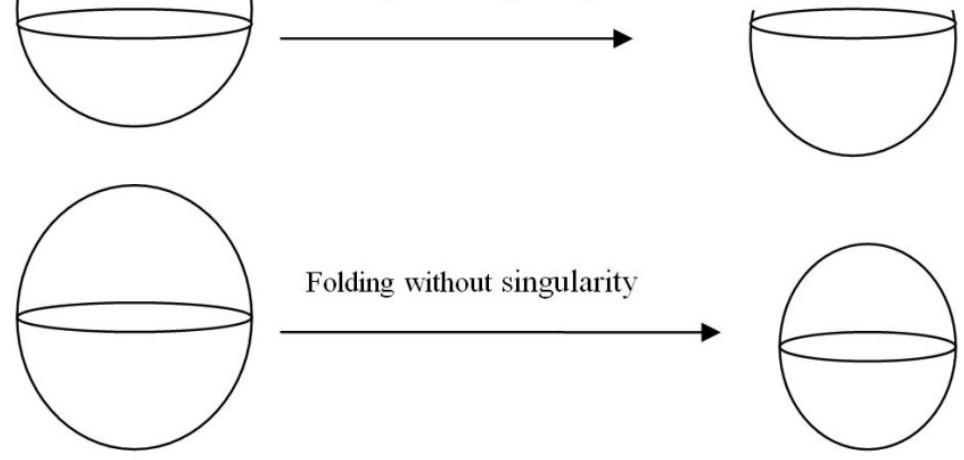

Folding without singularity

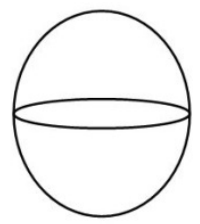


Figure 1

Corollary 3.2 The homology group of the limit of folding and unfolding of a manifold which is homeomorphic to $S^{2}, n>2$ is the identity group.

Theorem 3.3 The homology group $H_{n}(T)$ of any folding of $T$ is either isomorphic to $\mathrm{Z}, \mathrm{Z} \times \mathrm{Z}$ or identity group.

Proof. First, for folding with singularity of $T$ as in figure 2(a) then $H_{0}(F(T)) \approx \mathrm{Z}, H_{1}(F(T)) \approx \mathrm{Z}$ and for $n \geq 2, H_{n}(F(T))=0$. Also, folding without singularity of $T$ is a manifold homeomorphic to $T$ as in figure $2\left(\right.$ b) and so $H_{0}(F(T)) \approx \mathrm{Z}, \quad H_{1}(F(T))=\mathrm{Z} \times \mathrm{Z}, \quad H_{2}(F(T)) \approx \mathrm{Z}$, and $H_{n}(F(T))=0$, for $n>2$.

Therefore any folding of $T$ is either isomorphic to $\mathrm{Z}, \mathrm{Z} \times \mathrm{Z}$ or identity group.

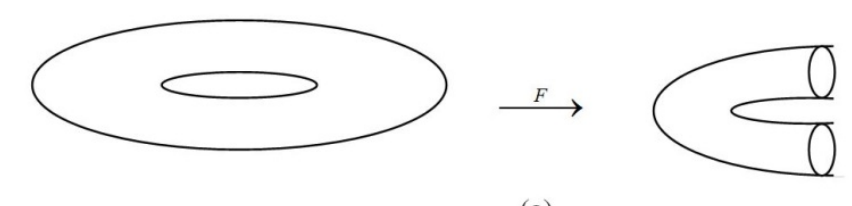

(a)

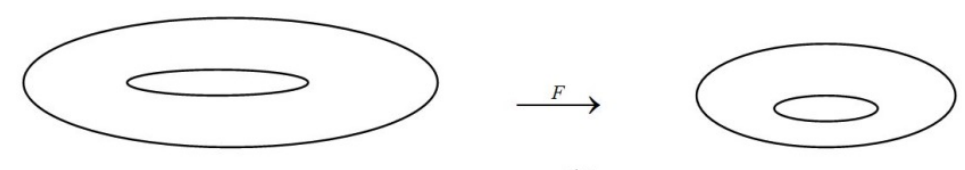

(b)

Figure 2

Corollary 3.4 The homology group of the limit of folding and unfolding of a manifold which is homeomorphic to $T, n>2$ is the identity group.

Theorem 3.5 If $F_{i}: S_{1}^{2} \vee S_{2}^{2} \rightarrow S_{1}^{2} \vee S_{2}^{2}, i=1,2$ are two types of folding such that $F_{i}\left(S_{j}^{2}\right)=S_{i}^{2}, j=1,2$. Then, $H_{n}\left(\lim _{n \rightarrow \infty} F_{i_{n}}\left(S_{1}^{2} \vee S_{2}^{2}\right)\right)$ is isomorphic to $\mathrm{Z}$ or identity group.

Proof. If $F_{i}: S_{1}^{2} \vee S_{2}^{2} \rightarrow S_{1}^{2} \vee S_{2}^{2}, i=1,2$ are two types of foldings such that $F_{i}\left(S_{j}^{2}\right)=S_{i}^{2}, j=1,2$, then $\lim _{n \rightarrow \infty} F_{i_{n}}\left(S_{1}^{2} \vee S_{2}^{2}\right)=S_{i}^{2}$ as in Figure (3). Thus, $H_{n}\left(\lim _{n \rightarrow \infty} F_{i_{n}}\left(S_{1}^{2} \vee S_{2}^{2}\right)\right)=H_{n}\left(S_{i}^{2}\right)$. Therefore, $H_{n}\left(\lim _{n \rightarrow \infty} F_{i_{n}}\left(S_{1}^{2} \vee S_{2}^{2}\right)\right)$ is isomorphic to $\mathrm{Z}$ or identity group.
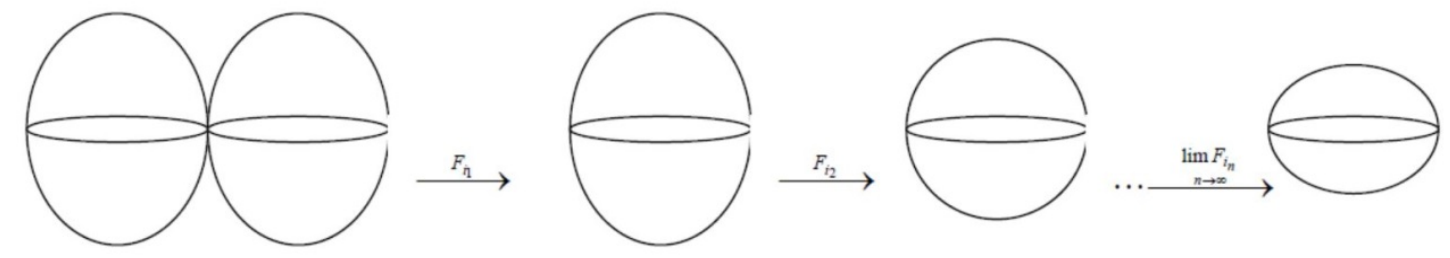

Figure 3

Theorem 3.6 If $F_{i}: T_{1} \vee T_{2} \rightarrow T_{1} \vee T_{2}, i=1,2$ are two types of foldings such that $F_{i}\left(T_{j}\right)=T_{i}, j=1,2$. Then $H_{n}\left(\lim _{n \rightarrow \infty} F_{i_{n}}\left(T_{1} \vee T_{2}\right)\right)$ is isomorphic to $\mathrm{Z}, \mathrm{Z} \times \mathrm{Z}$ or identity group, for all $n$. 
Proof. If $F_{i}: T_{1} \vee T_{2} \rightarrow T_{1} \vee T_{2}, i=1,2$ are two types of foldings such that $F_{i}\left(T_{j}\right)=T_{i}, j=1,2$, then $\left.\lim _{n \rightarrow \infty} F_{i_{n}}\left(T_{1} \vee T_{2}\right)\right)=T_{i} \quad$ as $\quad$ in $\quad$ Figure $\quad 4$, thus $\quad H_{n}\left(\underset{n \rightarrow \infty}{\lim F_{i_{n}}}\left(T_{1} \vee T_{2}\right)\right)=H_{n}\left(T_{i}\right) \quad$, since $H_{0}\left(T_{i}\right) \approx \mathrm{Z}, H_{1}\left(T_{i}\right) \approx \mathrm{Z} \times \mathrm{Z}, H_{2}\left(T_{i}\right) \approx \mathrm{Z}$, and if $k>2, H_{n}\left(T_{i}\right) \approx 0$ therefore, $H_{n}\left(\lim _{n \rightarrow \infty} F_{i_{n}}\left(T_{1} \vee T_{2}\right)\right)$ is isomorphic to $\mathrm{Z}, \mathrm{Z} \times \mathrm{Z}$ or identity group.

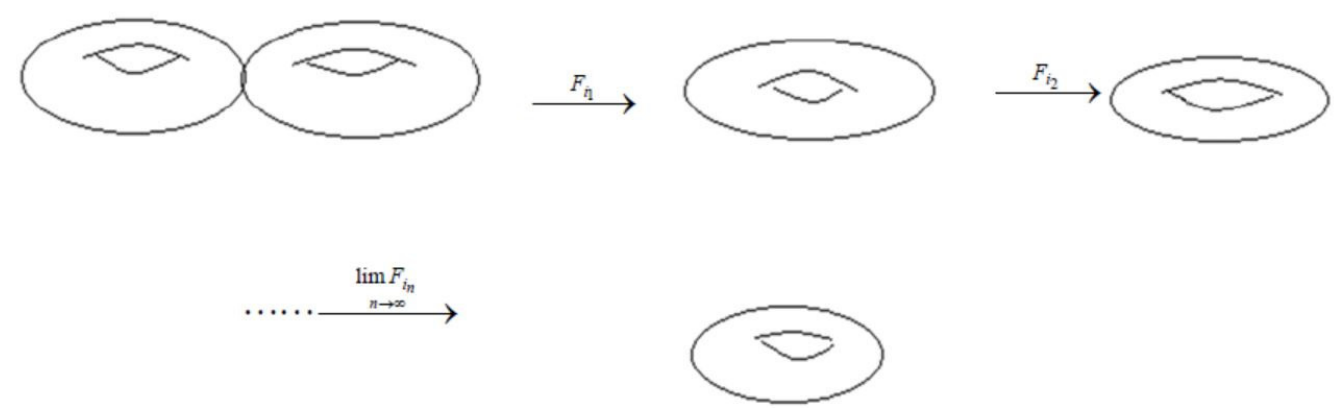

Figure 4

Theorem 3.7 If $F: S_{1}^{2} \vee S_{2}^{2} \rightarrow S_{1}^{2} \vee S_{2}^{2}$ is a folding by cut such that $F\left(S_{i}^{2}\right) \neq S_{i}^{2}$, for $i=1,2$. Then $H_{n}\left(\lim _{n \rightarrow \infty} F_{n}\left(S_{1}^{2} \vee S_{2}^{2}\right)\right)$ is isomorphic to identity group, for all $n>0$.

Proof. Consider $F\left(S_{i}^{2}\right) \neq S_{i}^{2}$, for $i=1,2$, then we have the following:

If $\lim _{n \rightarrow \infty} F_{n}\left(S_{1}^{2} \vee S_{2}^{2}\right)$ as in Figure 5, then $H_{n}\left(\lim _{n \rightarrow \infty} F_{n}\left(S_{1}^{2} \vee S_{2}^{2}\right)\right)=0$, forall $n>0$, therefore $H_{n}\left(\lim _{n \rightarrow \infty} F_{n}\left(S_{1}^{2} \vee S_{2}^{2}\right)\right)$ is isomorphic to identity group, for all $n>0$.
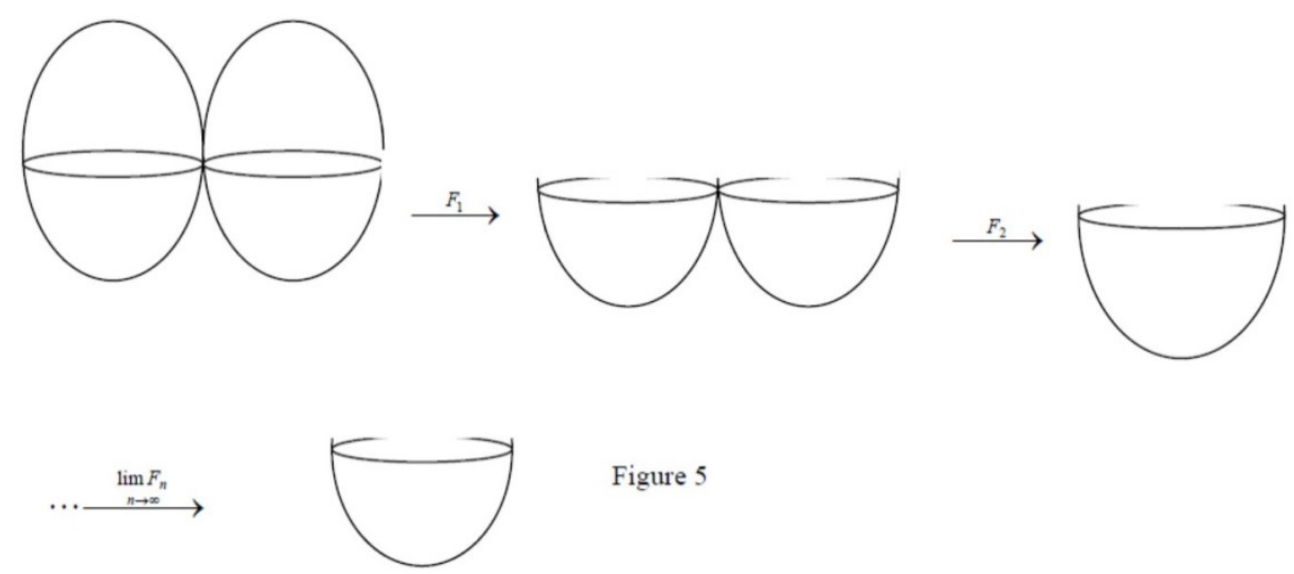

Theorem 3.8 If $F_{i}: S_{1}^{2} \vee S_{2}^{2} \rightarrow S_{1}^{2} \vee S_{2}^{2}, i=1,2$ are two types of foldings such that $F_{i}\left(S_{i}^{2}\right)=S_{i}^{2}, F_{j}\left(S_{i}^{2}\right) \neq S_{i}^{2}, j=1,2, i \neq j$. Then $H_{n}\left(\lim _{n \rightarrow \infty} F_{i_{n}}\left(S_{1}^{2} \vee S_{2}^{2}\right)\right)$ is isomorphic to $z$ or identity group, for all $n$.

Proof. Since $F_{i}\left(S_{i}^{2}\right)=S_{i}^{2}, F_{j}\left(S_{i}^{2}\right) \neq S_{i}^{2}, j=1,2, i \neq j$, we have the following: 
If $\lim _{n \rightarrow \infty} F_{i_{n}}\left(S_{1}^{2} \vee S_{2}^{2}\right)=S_{i}^{2}$ as in Figure 6. Thus, $H_{n}\left(\lim _{n \rightarrow \infty} F_{i_{n}}\left(S_{1}^{2} \vee S_{2}^{2}\right)\right)=H_{n}\left(S_{i}^{2}\right)$, therefore $H_{n}\left(\lim _{n \rightarrow \infty} F_{i_{n}}\left(S_{1}^{2} \vee S_{2}^{2}\right)\right)$ is isomorphic to $\mathrm{Z}$ or identity group, for all $n$.
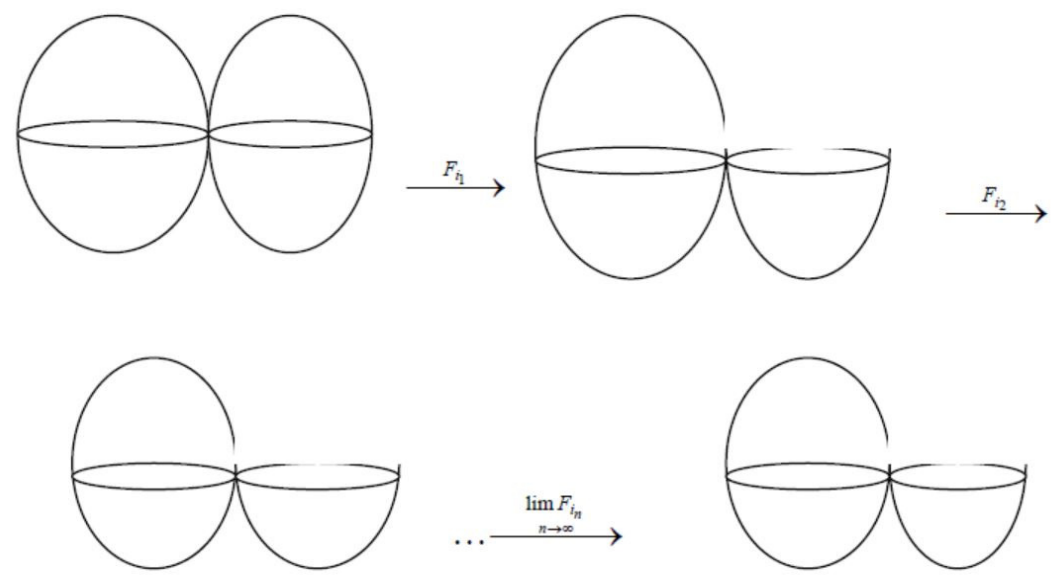

Figure 6

Theorem 3.9 If $F: T_{1} \vee T_{2} \rightarrow T_{1} \vee T_{2}$ is a folding by cut such that $F\left(T_{i}\right) \neq T_{i}$, for $i=1,2$. Then $H_{n}\left(\lim F_{n}\left(T_{1} \vee T_{2}\right)\right)$ is isomorphic to $\mathrm{Z}, \mathrm{Z} \times \mathrm{Z}$ or identity group, for all $n>0$.

Proof. Consider $F\left(T_{i}\right) \neq T_{i}$, for $i=1,2$, then we have the following:

If $\lim _{n \rightarrow \infty} F_{n}\left(T_{1} \vee T_{2}\right)=S_{1}^{1} \vee S_{2}^{1} \quad$ as $\quad$ in $\quad$ Figure $\quad 7(\mathrm{a})$, then $H_{n}\left(\lim _{n \rightarrow \infty} F_{n}\left(T_{1} \vee T_{2}\right)\right) \approx H_{n}\left(S_{1}^{2}\right) \times H_{n}\left(S_{2}^{2}\right) \quad$, so $H_{n}\left(\lim _{n \rightarrow \infty} F_{n}\left(T_{1} \vee T_{2}\right)\right) \approx \mathrm{Z} \times \mathrm{Z}$, or 0 for all $n>0$.

Also, if $\lim _{n \rightarrow \infty} F_{n}\left(T_{1} \vee T_{2}\right)$ as in Figure $7(\mathrm{~b})$, then $H_{n}\left(\lim _{n \rightarrow \infty} F_{n}\left(T_{1} \vee T_{2}\right)\right) \approx 0$, for all $n>0$. Moreover, if $\lim _{n \rightarrow \infty} F_{n}\left(T_{1} \vee T_{2}\right)$ as in Figure $7(\mathrm{c})$, then $H_{n}\left(\lim _{n \rightarrow \infty} F_{n}\left(T_{1} \vee T_{2}\right)\right) \approx \mathrm{Z}$, or 0 for all $n>0$. Therefore, $H_{n}\left(\lim _{n \rightarrow \infty} F_{n}\left(T_{1} \vee T_{2}\right)\right)$ is isomorphic to $\mathrm{Z}, \mathrm{Z} \times \mathrm{Z}$ or identity group, for all $n>0$. 


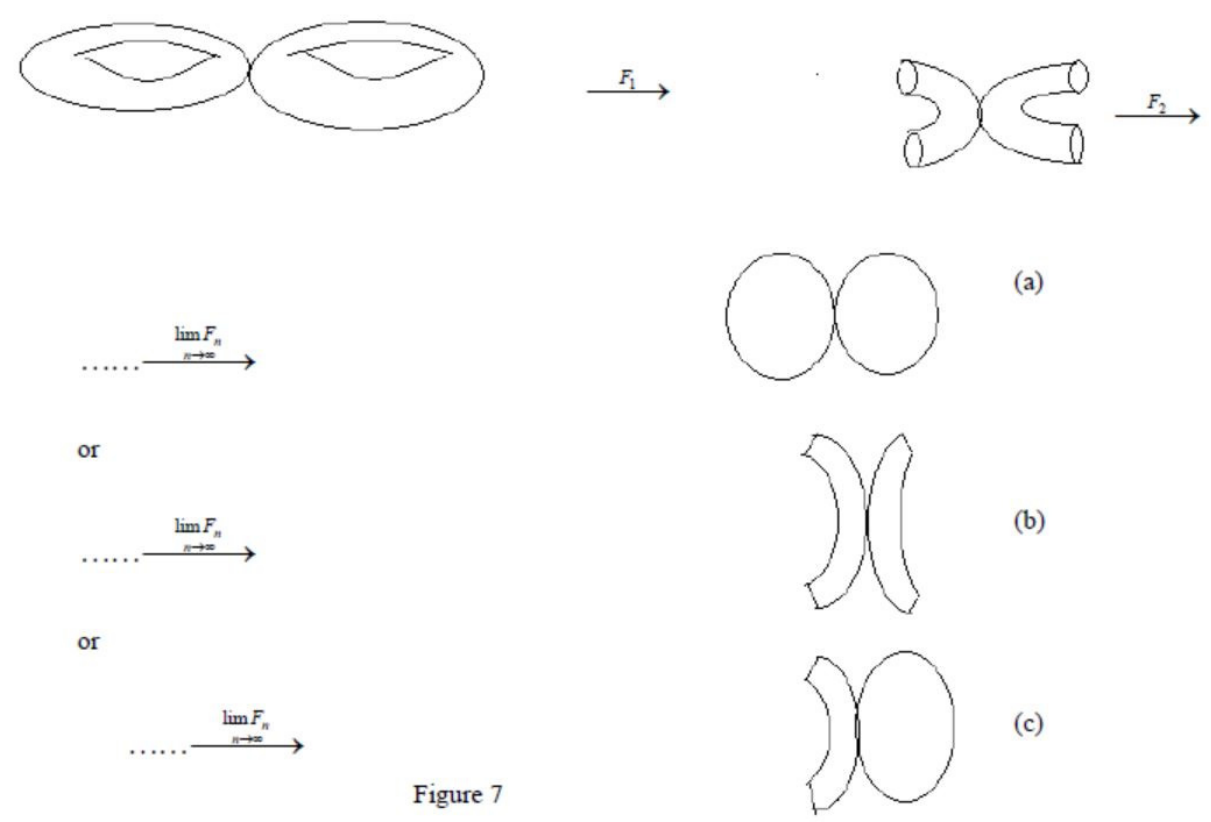

Theorem 3.10 If $F_{i}: T_{1} \vee T_{2} \rightarrow T_{1} \vee T_{2}, i=1,2$ are two types of foldings such that $F_{i}\left(T_{i}\right)=T_{i}, F_{j}\left(T_{i}\right) \neq T_{i}, j=1,2, i \neq j$. Then $H_{n}\left(\lim F_{i_{n}}\left(T_{1} \vee T_{2}\right)\right)$ is isomorphic to $(\mathrm{Z} \times \mathrm{Z}) \times \mathrm{Z}$, $\mathrm{Z} \times \mathrm{Z}$ or identity group, for all $n>0$.

Proof. If $F_{i}: T_{1} \vee T_{2} \rightarrow T_{1} \vee T_{2}, i=1,2$ are two types of foldings such that $F_{i}\left(T_{i}\right)=T_{i}, F_{j}\left(T_{i}\right) \neq T_{i}, j=1,2, i \neq j$, we have the following:

If $\lim _{n \rightarrow \infty} F_{i_{n}}\left(T_{1} \vee T_{2}\right)=T_{i} \vee S_{i}^{1}$ as in Figure 8(a). Then, $H_{n}\left(\lim _{n \rightarrow \infty} F_{i_{n}}\left(T_{1} \vee T_{2}\right)\right)=H_{n}\left(T_{i} \vee S_{i}^{1}\right) \approx(\mathrm{Z} \times \mathrm{Z}) \times \mathrm{Z}$ or 0 , for all $n>0$.

Also, if $H_{n}\left(\lim _{n \rightarrow \infty} F_{i_{n}}\left(T_{1} \vee T_{2}\right)\right)=H_{n}\left(T_{i}\right) \approx(\mathrm{Z} \times \mathrm{Z})$ or 0 , for all $n>0$ as in Figure $8(\mathrm{~b})$. Therefore, $H_{n}\left(\lim _{n \rightarrow \infty} F_{i_{n}}\left(T_{1} \vee T_{2}\right)\right)$ is isomorphic to $(\mathrm{Z} \times \mathrm{Z}) \times \mathrm{Z}, \mathrm{Z} \times \mathrm{Z}$ or identity group, for all $n>0$. 

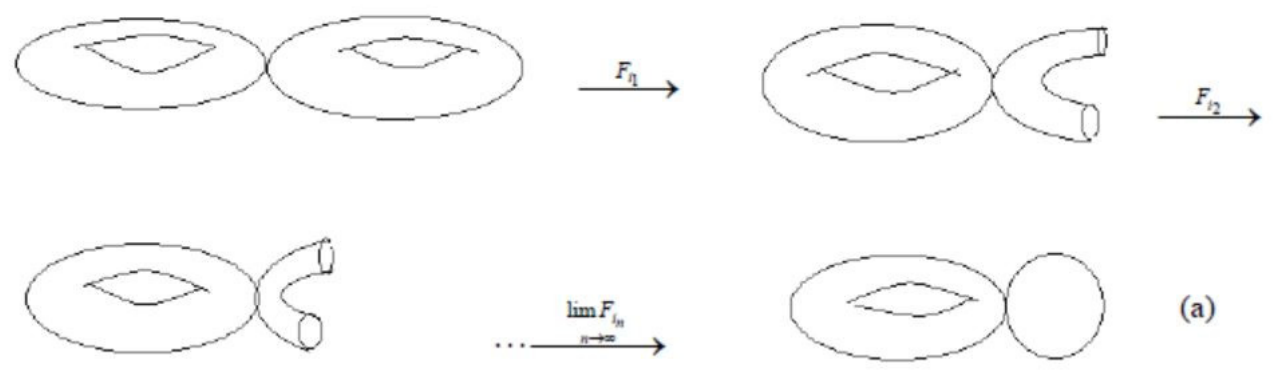

(a)

or
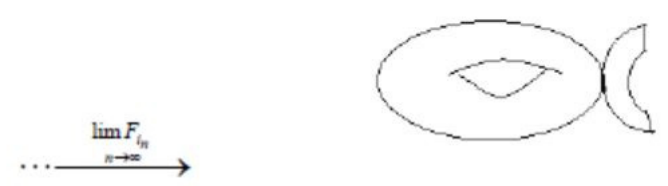

(b)

Figure 8

Lemma 3.11 Let $M_{1}, M_{2}$ be two disjoint spheres in $R^{3}$. Then there is unfolding unf $: M_{1} \cup M_{2} \rightarrow M_{1}^{\prime} \cup M_{2}^{\prime}$ which induces unfolding $\overline{u n f}: H_{n}\left(M_{1} \cup M_{2}\right) \rightarrow H_{n}\left(M_{1}^{\prime} \bigcup M_{2}^{\prime}\right)$ such that

(1) $\overline{u n f}\left(H_{0}\left(M_{1} \cup M_{2}\right)\right) \approx \mathrm{Z}$,

(2) $\overline{\operatorname{unf}}\left(H_{1}\left(M_{1} \cup M_{2}\right)\right) \approx 0$,

(3) $\overline{u n f}\left(H_{2}\left(M_{1} \cup M_{2}\right)\right) \approx \mathrm{Z} \oplus \mathrm{Z}$,

(4) $\overline{u n f}\left(H_{n}\left(M_{1} \cup M_{2}\right)\right) \approx 0$, for $n>2$.

Proof. Let unf: $M_{1} \cup M_{2} \rightarrow M_{1}^{\prime} \cup M_{2}^{\prime}$ be unfolding such that $\operatorname{unf}\left(M_{1} \cup M_{2}\right)=\operatorname{unf}\left(M_{1}\right) \vee \operatorname{unf}\left(M_{2}\right)$ as in figure 9 , thus we get the induced unfolding $\overline{u n f}: H_{n}\left(M_{1} \cup M_{2}\right) \rightarrow H_{n}\left(M_{1}^{\prime} \cup M_{2}^{\prime}\right)$ such that $H_{n}\left(u n f\left(M_{1} \cup M_{2}\right)\right)=H_{n}\left(u n f\left(M_{1}\right) \vee \operatorname{unf}\left(M_{2}\right)\right)$. Now, for $n=0, \quad \overline{u n f}\left(H_{0}\left(M_{1} \cup M_{2}\right)\right)=H_{0}\left(\operatorname{unf}\left(M_{1} \cup M_{2}\right)\right) \approx \mathrm{Z}$. And $\quad$ if $\quad n=1, \quad \overline{\operatorname{unf}}\left(H_{1}\left(M_{1} \cup M_{2}\right)\right)=H_{1}\left(\operatorname{unf}\left(M_{1} \cup M_{2}\right)\right) \approx 0 \quad$. Also, if $\quad n=2$, $\overline{\operatorname{unf}}\left(H_{2}\left(M_{1} \cup M_{2}\right)\right)=H_{2}\left(\operatorname{unf}\left(M_{1} \cup M_{2}\right)\right) \approx H_{2}\left(u n f\left(M_{1}\right)\right) \oplus H_{2}\left(\operatorname{unf}\left(M_{2}\right)\right)$. Since $\quad H_{2}\left(\operatorname{unf}\left(M_{i}\right) \approx Z, i=1,2 \quad\right.$ it follows that $\overline{\operatorname{unf}}\left(H_{2}\left(M_{1} \cup M_{2}\right)\right) \approx \mathrm{Z} \oplus \mathrm{Z}$.

Moreover, if $n \geq 3$, it follows that $H_{n}\left(u n f\left(M_{i}\right) \approx 0, i=1,2\right.$. Thus $\overline{u n f}\left(H_{n}\left(M_{1} \cup M_{2}\right)\right) \approx 0$, for $n>2$. 


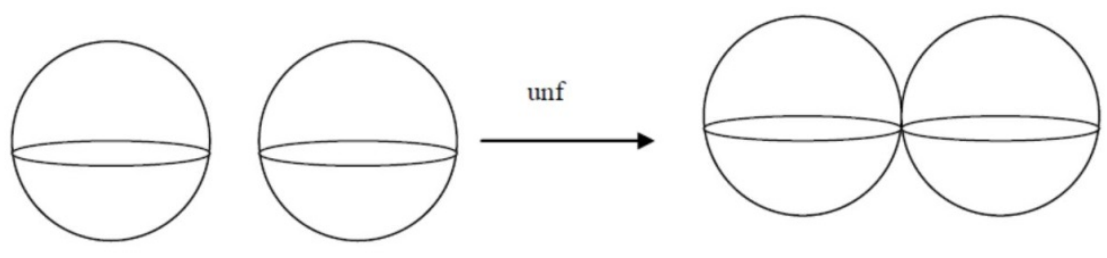

Figure 9

Theorem 3.12 Let $D_{k}$ be the disjoint union of $k$-discs on the sphere $S^{2}$. Then there is unfolding unf $:\left(S^{2}-D_{k}\right) \rightarrow S^{2}$ such that $H_{2}\left(\lim _{m \rightarrow \infty}\left(u n f_{m}\left(S^{2}-D_{k}\right)\right)\right) \approx \mathrm{Z}$.

Proof: Let $D_{k}$ be the disjoint union of $k$-discs on the sphere $S^{2}$. Then, we can define a sequence of unfolding

$$
\begin{aligned}
& u n f_{1}: S^{2}-D_{k} \rightarrow M_{1}, S^{2}-D_{k} \subseteq M_{1} \subseteq S^{2} \\
& u n f_{2}: M_{1} \rightarrow M_{2}, M_{1} \subseteq M_{2} \subseteq S^{2} \\
& \vdots \\
& u_{u n f}: M_{m-1} \rightarrow M_{m}, M_{1} \subseteq M_{2} \subseteq \ldots \subseteq M_{m-1} \subseteq M_{m} \subseteq S^{2}
\end{aligned}
$$

Such that $\quad \lim _{m \rightarrow \infty} u n f_{m}\left(S^{2}-D_{k}\right)=S^{2} \quad$ as in figure $10 \quad$ for $\quad k=2$. Hence

$$
H_{2}\left(\lim _{m \rightarrow \infty}\left(u n f_{m}\left(S^{2}-D_{k}\right)\right)\right)=H_{2}\left(S^{2}\right)=\mathrm{Z}
$$

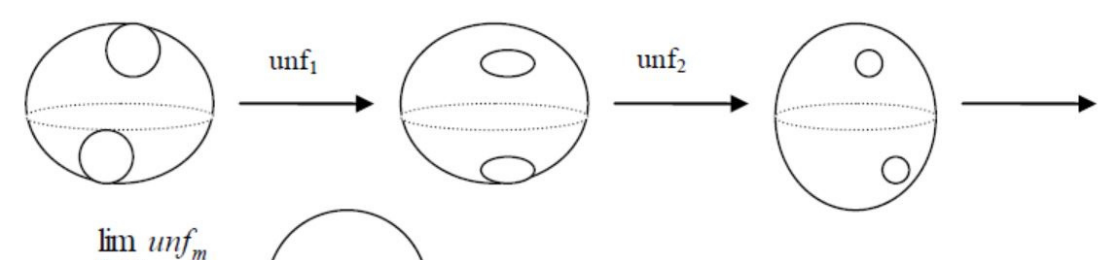

Figure 10

\section{Conclusion.}

Folding and unfolding problems have been implicit for long time, but have not been studied extensively in the mathematical literature until recently .Over the past few years; there has been a surge of interest in these problems in discrete and computational geometry. This paper gives the folding and unfolding of some types of manifolds, which are determined by their homology group and we discussed the homology group of the limit of folding and unfolding on a wedge sum of 2-manifolds.

The main results can be similarly extended to some other some geometric shapes such as polyhedra .The problems lies: Can all convex polyhedra be edge-unfolded, and can all polyhedra be generally unfolded?

\section{Acknowledgement}

The authors are thankful to the referees for a careful reading of the paper and for valuable comments and suggestions.

\section{References}

Abu-Saleem, M. (2007). Some geometric transformations on manifolds and their algebraic structures, Ph.D.Thesis, University of Tanta, Egypt. 
Abu-Saleem, M. (2010). Folding and unfolding of manifolds and their fundamental groups. Int. J. Contemp.Math.Sciences, 5(1), 1-9.

Abu-Saleem, M. (2010). Homology group on manifolds and their foldings. Tamkang Journal of Mathematics, 41(1), 31-38.

El-Ghoul, M. (1988). Unfolding of Riemannian manifold. Commun.Fac.Sci.University of Ankara Series A, 37, $1-4$.

El-Ghoul, M. (1990). Homology group and the deformation retract of a compact manifold and its foldings. $J$. Inst. Math. and Comp.Sci. Math.Ser., India, 3(1), 51-54.

El-Ghoul, M., El-Ahmady, A.E., Rafat, H., \& M.Abu-Saleem (2006). The fundamental group of the connected sum of manifolds and their foldings. In. J.Pure and applied math, 29, 385-392.

El-Ghoul, M., El-Ahmady, A.E., Rafat, H., \& M.Abu-Saleem (2011). Effects of foldings on free product of fundamental groups. International Journal of Math.Combin., 2, 29-39.

El-Ghoul, M., Nada, S.I., \& Abo Elanin, R.M. (2009). On the folding of rings. International journal of algebra, 3, 475-482.

El-Kholy, E.M. \& El-Esawy, A. (2005). Graph folding of some special graphs. J.Math.Statistics, 1, 66-70. http://dx.doi.org/10.3844/jmssp.2005.66.70

El-Kholy, E.M. (1981). Isometric and Topological folding of manifolds, Ph.D.Thesis, university of Southampton UK.

Giblin, P.J. (1977). Graphs, surfaces and homology, an introduction to algebraic topology. Champman and Hall Ltd, London.

Hatcher, A. (2001). Algebraic Topology, http://www.math.cornell.edu/hatcher.

Massey,W. S. (1967). Algebraic Topology, an introduction. Harcourt, Brace \& world, Inc., NewYork U.S.A.

Robertson, S. A. (1977). Isometric folding of Riemannian manifolds. Proc. Roy. Soc. Edinburgh, 77, 275-289.

\section{Copyrights}

Copyright for this article is retained by the author(s), with first publication rights granted to the journal.

This is an open-access article distributed under the terms and conditions of the Creative Commons Attribution license (http://creativecommons.org/licenses/by/3.0/). 